\title{
Modeling of Objects Using Conic Splines
}

\author{
Muhammad Sarfraz ${ }^{1}$, Malik Z. Hussain ${ }^{2}$, Munazah Ishaq ${ }^{2}$ \\ ${ }^{1}$ Department of Information Science, Kuwait University, Adailiya Campus, Safat, Kuwait; ${ }^{2}$ Department of Mathematics, University \\ of the Punjab, Lahore, Pakistan. \\ Email: prof.m.sarfraz@gmail.com
}

Received 2013

\begin{abstract}
This paper contributes towards modeling for the designing of objects in the areas of Computer Graphics (CG), Computer-Aided Design (CAD), Computer-Aided Manufacturing (CAM), and Computer-Aided Engineering (CAE). It provides a modeling technique for the designing of objects. The model is based on a conic-like curve (rational quadratics) method and provides an extra degree of freedom to the user to fine tune the shape of the design to the satisfactory level. The 2D curve model has then been extended for the designing of 3D objects to produce fancy objects. The scheme has been also extended to automate the degree of freedom when a reverse engineering is required for images of the objects. A heuristic technique of genetic algorithm is applied to find optimal values of shape parameters in the description of conics.
\end{abstract}

Keywords: Computer Graphics; CAD; CAM; CAE; Splines

\section{Introduction}

Object modeling is an important area of Computer Graphics (CG), Computer-Aided Design (CAD), ComputerAided Manufacturing (CAM), and Computer-Aided Engineering (CAE). No matter, it is designing of an aircraft or an outline of a font, most of the objects are being designed through computers. This area of study has attracted many scientists to work for different industries including engineering works, medical, entertainment, etc. Since the advent of computers, numerous authors have discovered various methodologies in various disciplines.

This research is mainly concerned for the designing of 2D objects composed of curve outlines and then it is extended for the 3D objects based on rectangular domain. It is in continuation of the spline introduced in [27]. Spline curves and surfaces [1-27] play significant role in the construction and re-construction of objects. The splines including Beta-splines [18-24], Nu-splines [5], and weighted Nu-splines [5] make a good contribution to CG, CAD, CAM, CAE, and Geometric Modeling. A reasonable amount of literature is available (see [1-27]) on this subject. Various other forms of splines are also available in the literature. For example, conic presentation can be found in [10-11], cubic flavor can be seen in [5,7-8,12], and B-splines work can be searched in [14-16]. The usefulness of rational splines can also not be denied, much of work has been done in this direction. For brevity, the reader is referred to $[1-2,4,13]$.
In [14], rational cubic spline, with derivatives based on control points, was discovered. This spline method has the feature of local interval shape control. This paper has used a different alternative interpolant which is rational quadratic in its description, but serves in the same manner as the rational cubic spline [14]. It is computationally economical and achieves equivalently fine results. All the features of cubic or rational cubic splines can be characterized by this quadratic method. Particularly, it holds the feature of local interval shape control as in [14]. Thus, any type of plane or space curve can be designed with having a strong control over the intervals of control points. In addition, the scheme has the following properties which may lead to a more useful approach to curve design in CAD, CAM, CAE, Computer Graphics, and Geometric Modeling:

(1) The curve has $C^{1}$ continuity.

(2) This scheme is rational quadratic and hence is simpler than the rational cubic scheme in [14].

(3) This scheme is an extended work done in [27].

(4) The method is local, i.e. the interval tension applied by shape control parameters will affect very small neighborhood of interval.

(5) This scheme is as suitable as any cubic or rational cubic method for space curves and hence can be generalized to surfaces.

(6) Any part of the rational quadratic spline method is a conic and can be made straight line using the same interpolant. 
(7) The rational quadratic curve scheme is extendable to its rational bi-quadratic counterpart for the designing or re-designing of 3D objects.

The paper, in addition to curve design method, also proposes a surface design scheme. This has been achieved by extending the proposed rational quadratic curve scheme to its rational bi-quadratic counterpart. The presentation of surface model is mainly a tensor product surface model. It is simple in its description and useful for the designing of 3D objects.

The outline of the remainder of the paper is as follows. The mention of the piecewise rational quadratic interpolant is made in Section 2. This section describes the parametric rational quadratic spline interpolation scheme. Analysis of the design curve, regarding various geometric features, is made in Section 3. The effect of shape control analysis is described in Section 4. The construction of 3D surfaces has been briefly explained pictorially in Section 5. Section 6 concludes the paper.

\section{Design Curve}

In this section, piecewise rational quadratic functions are presented to be used for curve designing and fitting. The rational quadratic is targeted to provide $C^{1}$ continuity. It is also required that the quadratic curve presentation is extended towards its surface designing counterpart.

\subsection{Rational Quadratic Function}

The objective of this research is to provide design curve scheme which

- is composed of conics like curve (see Figure 1) the following:

$$
F(\theta)=\frac{(1-\theta)^{2} b_{0}+2 \theta(1-\theta) w b_{1}+\theta^{2} b_{2}}{(1-\theta)^{2}+2 \theta(1-\theta) w+\theta^{2}}
$$

- interpolates the data points

- possesses ideal geometric properties like convex hull and variation diminishing properties

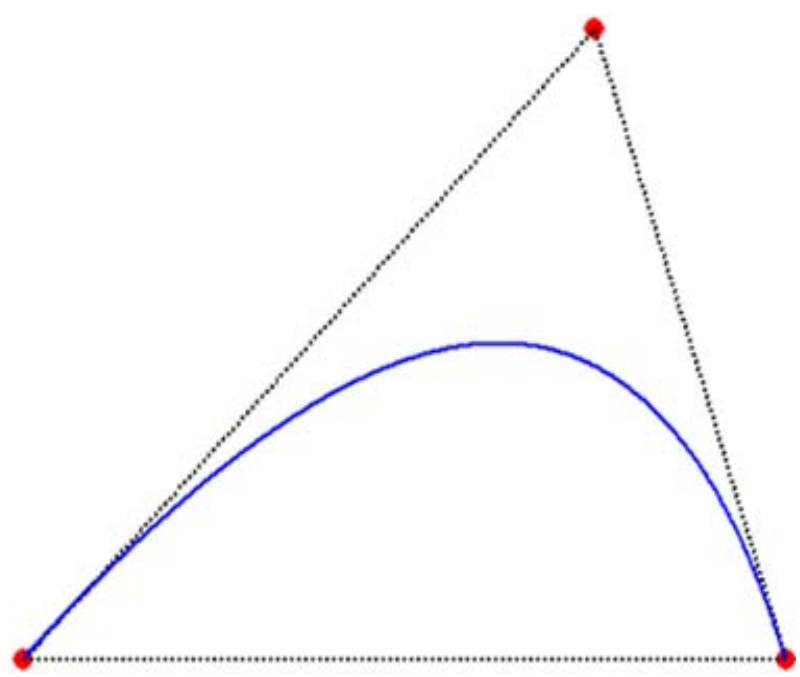

Figure 1. Demonstration of rational quadratic function.

- can handle the inflection points like cubic or rational cubic spline curves

- provides freedom to the designer for further shape control

- is reasonably smooth

\subsection{Rational Quadratic Spline}

Let

$$
F_{i} \in R^{m}, i=0,1, \ldots . n,
$$

be a given set of data points at the distinct knots $t_{i} \in R$, with unit interval spacing. Let

$$
t_{i}^{*}=\left(t_{i}+t_{i+1}\right) / 2 \text {, and } h_{i}=t_{i+1}-t_{i} .
$$

Also let

$$
U_{i}, W_{i}, V_{i} \in R^{m}, i=0,1, \ldots . n-1,
$$

be the control points. Then, we define a parametric piecewise rational quadratic function $P: R \rightarrow R^{m}$, composed with two conic representations (see Figures 2(a) and (b)) of the form (1), as follows:
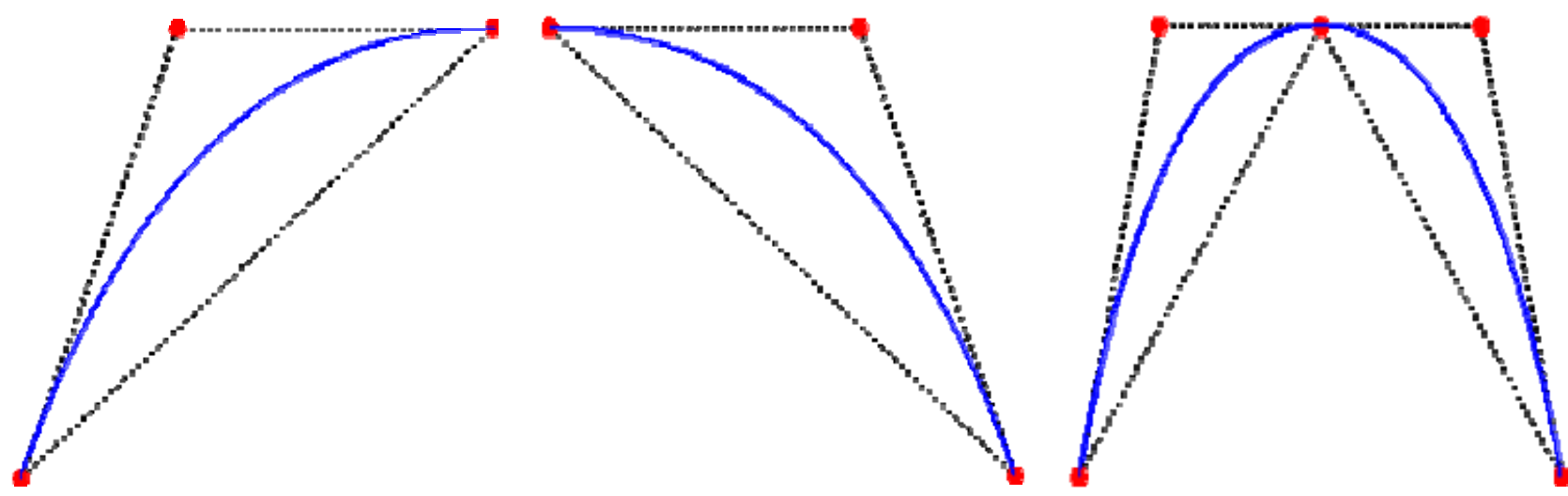

Figure 2. Rational quadratic representations: (a) Conic piece in (6), (b) Conic piece in (7), (b) Conic spline curve in (5). 


$$
P_{i}(t)=\left\{\begin{array}{l}
P_{1, i}(\theta), \theta \equiv \theta(t)=2\left(t-t_{i}\right) / h_{i}, t \in\left[t_{i}, t_{i}^{*}\right], \\
P_{2, i}(\vartheta), \vartheta \equiv \vartheta(t)=2\left(t-t_{i}^{*}\right) / h_{i}, t \in\left[t_{i}^{*}, t_{i+1}\right]
\end{array}\right.
$$

where

$$
P_{1, i}(t)=\frac{(1-\theta)^{2} F_{i}+\theta(1-\theta)^{2} \gamma_{i} U_{i}+\theta^{2} W_{i}}{(1-\theta)^{2}+\theta(1-\theta) \gamma_{i}+\theta^{2}}
$$

and

$$
P_{2, i}(t)=\frac{(1-\vartheta)^{2} W_{i}+\vartheta(1-\vartheta)^{2} \gamma_{i} V_{i}+\vartheta^{2} F_{i+1}}{(1-\vartheta)^{2}+\vartheta(1-\vartheta) \gamma_{i}+\vartheta^{2}}
$$

It can be seen that the rational quadratic curve (5) is $C^{0}$ as the followings hold:

$$
P_{1, i}\left(t_{i}^{*}\right)=P_{2, i}\left(t_{i}^{*}\right) \text { and } P_{i}\left(t_{i+}\right)=P_{i}\left(t_{i-}\right), \forall i \text {. }
$$

Let $P(t)$ be the curve over the given knot partition and passing through the given set of points (2). Let us denote the first derivatives at the knot position as:

$$
P^{(1)}\left(t_{i}\right)=D_{i}, \forall i
$$

Since $C^{0}$ curve is not ideal as far as smoothness is concerned, we need higher order of continuity. To obtain $C^{1}$ order of continuity, we need to impose the following constraints:

$$
\left.\begin{array}{l}
P^{(1)}\left(t_{i+}^{*}\right)=P^{(1)}\left(t_{i-}^{*}\right) \\
P^{(1)}\left(t_{i+}\right)=P^{(1)}\left(t_{i-}\right), \forall i
\end{array}\right\}
$$

These $C^{1}$ constraints, after some analysis and simplifications, yield the following:

and

$$
\left.\begin{array}{l}
U_{i}=F_{i}+\frac{h_{i} D_{i}}{2 \gamma_{i}} \\
V_{i}=F_{i+1}-\frac{h_{i} D_{i+1}}{2 \gamma_{i}}
\end{array}\right\}
$$

$$
W_{i}=\frac{U_{i}+V_{i}}{2}=\frac{F_{i}+F_{i+1}}{2}+\frac{h_{i}\left(D_{i}+D_{i+1}\right)}{4 \gamma_{i}}
$$

\section{Design Curve Analysis}

This section describes the parametric rational quadratic spline interpolation scheme. Analysis of the design curve, regarding parameterization, derivative estimation, shape parameters and various geometric features, is made in this section. It also demonstrates design curves with various practical examples.

\subsection{Parameterization}

Number of parameterization techniques can be found in literature for instance uniform parameterization, linear or chord length parameterization, parabolic parameterize- tion and cubic parameterization. In this paper, chord length parameterization is used to estimate the parametric value $t$ associated with each point. It can be observed that $\theta_{i}$ is in normalized form and varies from 0 to 1 . Consequently, in our case, $h_{i}$ is always equal to 1 .

\subsection{Estimation of Tangent Vectors}

A distance based choice of tangent vectors $D_{i}$ 's at $F_{i}$ 's is defined as:

$$
\left.\begin{array}{l}
D_{0}=2\left(F_{1}-F_{0}\right)-\left(F_{2}-F_{0}\right) / 2 \\
D_{n}=2\left(F_{n}-F_{n-1}\right)-\left(F_{n}-F_{n-2}\right) / 2 \\
D_{i}=a_{i}\left(F_{i}-F_{i-1}\right)-\left(1-a_{i}\right)\left(F_{i+1}-F_{i}\right), i=1,2, \ldots, n-1
\end{array}\right\}
$$

For close curves:

$$
\left.\begin{array}{l}
F_{-1}=F_{n-1}, F_{n+1}=F_{1} \\
D_{i}=a_{i}\left(F_{i}-F_{i-1}\right)-\left(1-a_{i}\right)\left(F_{i+1}-F_{i}\right), i=0,1, \ldots, n
\end{array}\right\}
$$

where

$$
a_{i}=\frac{\left|F_{i+1}-F_{i}\right|}{\left|F_{i+1}-F_{i}\right|+\left|F_{i}-F_{i-1}\right|}, i=0,1, \ldots, n
$$

\section{Shape Analysis}

This section is concerned about the shape design aspects to achieve a model curve. The parameters $\gamma_{i}$ may be used to control the shape of the curve. The parameters $\gamma_{i}$ are mainly meant to be used freely to control the shape of the curve. At the same time, for the convenient of the designer, it is also required that the ideal geometric properties of the curve are not lost. The geometric properties like variation diminishing, convex hull, and positivity are the ones which needed to be present in the description of the design curve.

One can see that each conic representation in (5-7) is of Bernstein Bezier form, provided the weight functions are positive. This is possible if the shape parameters are constrained as $\gamma_{i} \geq 0, \forall i$. Thus following the Bernstein Bezier theory, the pieces of curves $P_{1, i}(t)$ and $P_{2, i}(t)$ lie in the convex hulls of $\left\{F_{i}, U_{i}, W_{i}\right\}$ and $\left\{W_{i}, V_{i}, F_{i+1}\right\}$ respectively. They also follow the variation diminishing properties within their convex hulls. Similarly, due to $C^{1}$ imposition, the equations (8-10) lead to the followings:

- $P_{i}(t)$ lies in the convex hull of $\left\{F_{i}, U_{i}, W_{i}, V_{i}, F_{i+1}\right\}$, $\forall i$, see Figure 3 .

- $P_{i}(t)$ satisfies the variation diminishing property [22]. That is any straight line crossing the control polygon of $\left\{F_{i}, U_{i}, W_{i}, V_{i}, F_{i+1}\right\}$ does not cross the curve more than its control polygon. 


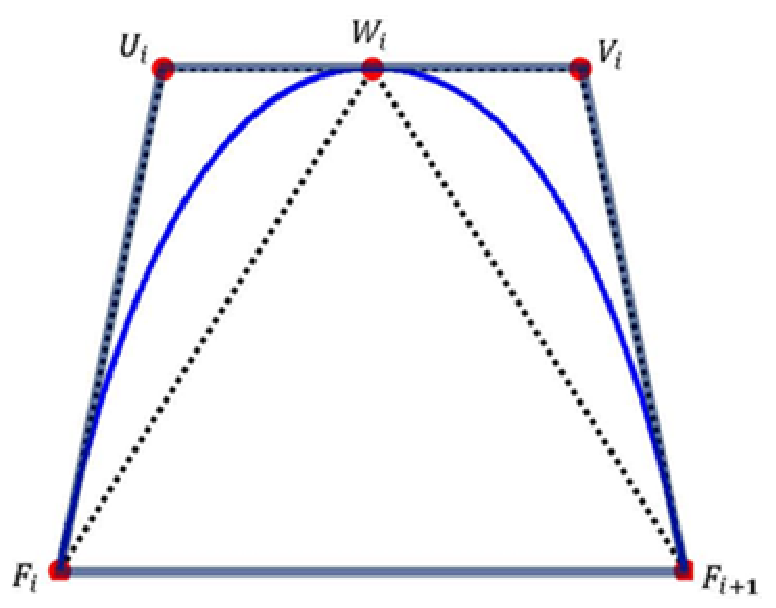

Figure 3. Convex Hull property.

An efficient algorithm, for generating an interpolating curve outline, which modifies its shape interactively according to the proposals described in above sections, has been implemented. The details of the algorithm are omitted due to fear of length of the paper. However, for an outline in Figure 6(a), the pictorial demonstration is made in Figure 6(b) to achieve a desired design of a character " $G$ ". A better designed conic spline (for the case of different values $\gamma_{i}^{\prime} \mathrm{s}$ in different intervals) has been fitted to a data (gray bullets) of character “G”.

\section{3D Modelling}

The 2D curve outline model, in Sections 2, 3, and 4, has been generalized and extended to build a 3D model which can have the provision of designing $3 \mathrm{D}$ objects. The details of the strategy in the construction of this model, due to fear of the length of the manuscript, have been left and will be presented somewhere else.

A demonstration of the default surface model is shown in Figure 7. Figure 7(a) shows that the first half of the surface and other half of the surface is shown in Figure 7(b). The default bi-quadratic surface (the composition of the two surfaces i.e. Figures 7(a) and (b)) is shown in Figure 7(c).
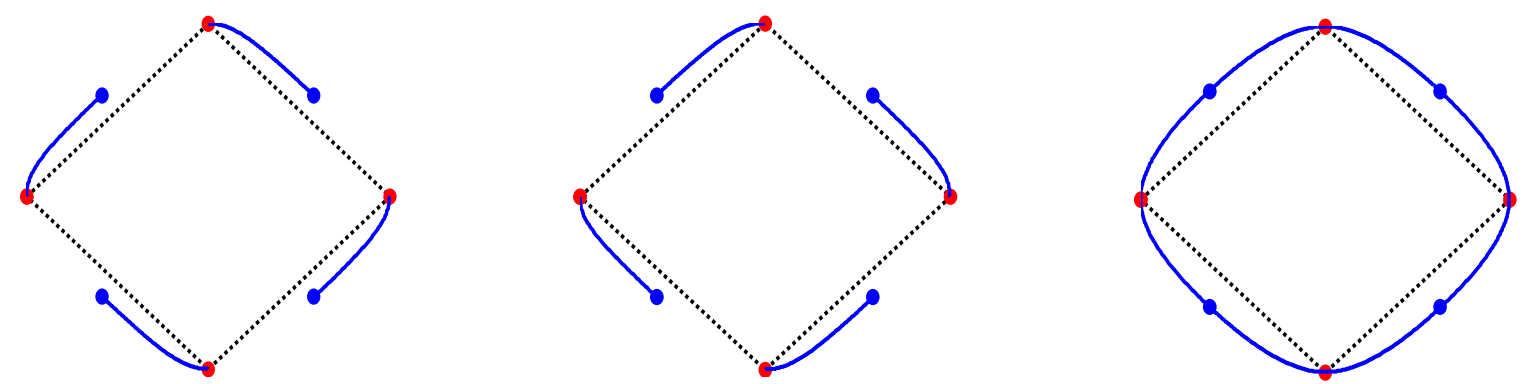

Figure 4. Rational quadratic spline (default curve case): (a) Rational Quadratic (6), (b) Rational Quadratic (7), (c) Rational Quadratic (5).

(a)

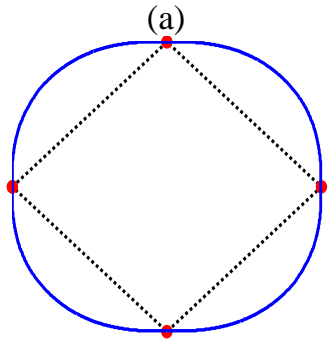

(d)

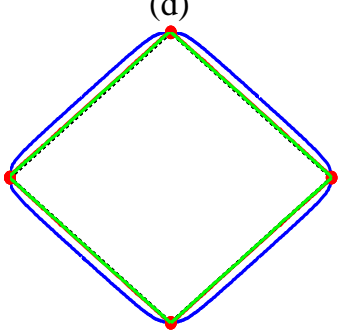

(b)

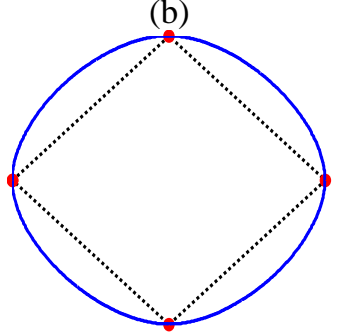

(c)

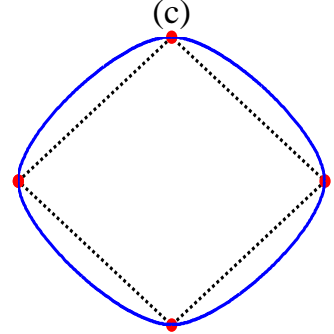

(e)

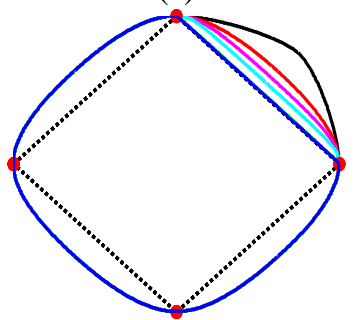

Figure 5. Rational quadratic spline: (a) Rational quadratic spline for $\gamma_{i}=1$, (b) Rational quadratic spline for $\gamma_{i}=2$, (c) Rational quadratic spline for $\gamma_{i}=3$, (d) Rational quadratic spline, with global shape control, for $\gamma_{i}=5,10$, and 50, (e) Rational quadratic spline, with local shape control, for $\gamma_{i}=1,2,3,5$, and 50 . 
Some more pictorial demonstrations are made as follows. A wire frame model, with $u$-lines is constructed, as the first step in the Figure 8(a) of a bottle. Secondly, $u v$-lines are drawn to provide a complete wire frame model (see Figure 8(b)). Thirdly, visibility detection method is used to provide a realistic view of the picture (see Figure 8(c)). Another feature built in the 3D model is the local shape control like in the 2D models demonstrated in Section 3.3. This feature can be seen in Figures $8(\mathbf{a}-\mathbf{c})$ at the neck of the bottle showing interval tension.

Another pictorial example of a vase is shown in Figure 9. The wire frame model is shown in Figure 9(a) and its shaded model is demonstrated in Figure 9(b). A variety of shape control has been applied at different places to achieve the shown model. (a)

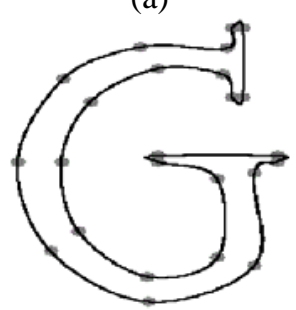

(b)

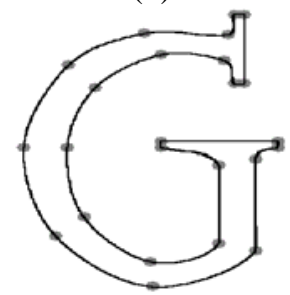

Figure 6. Rational quadratic spline: (a) Rational quadratic spline for $\gamma_{i}=2$, (b) Rational quadratic spline for varying values of $\gamma_{i}$ in different intervals. (a)

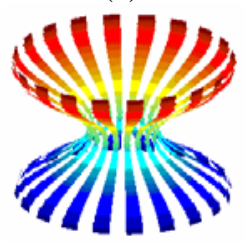

(b)

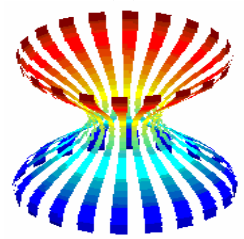

(c)

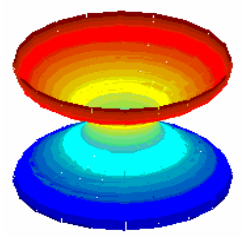

Figure 7. Rational bi-quadratic spline interpolant: (a) Rational bi-quadratic 1.(b) Rational bi-quadratic 2, (c) Rational bi-quadratic. (a)

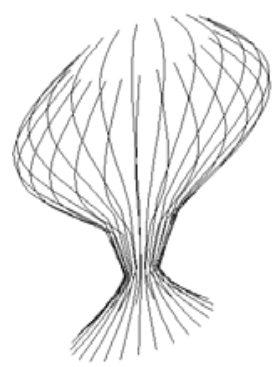

(b)

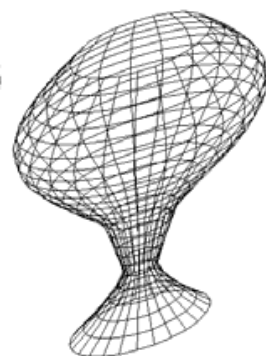

(c)

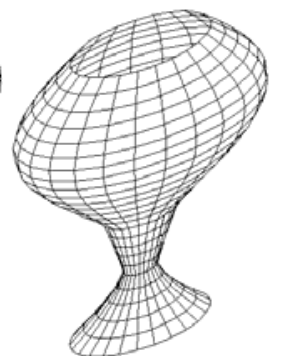

Figure 8. Rational bi-quadratic spline for the design of a bottle: (a) A wire frame model, with u-lines, (b) A wire frame model with uv-lines, (c) A wire frame model with uv-lines and hidden surface removal. (a)

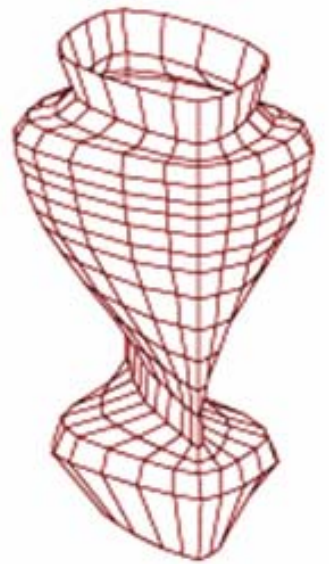

(b)

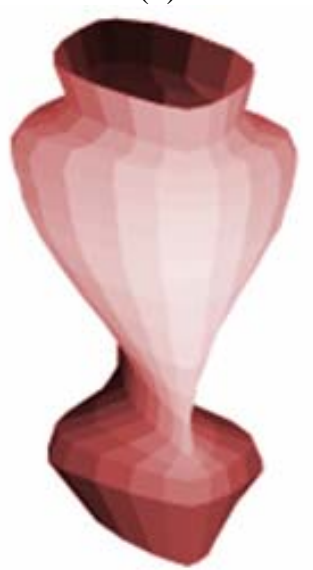

Figure 5. A bi-conic Spline model for a Vase: left one is a wire-frame model, right one is the shaded model.

\section{Conclusion and Future Work}

A shape controlling piecewise rational quadratic interpolation scheme, to design 2D and 3D objects, has been proposed. The scheme offers a possible and feasible way in which the shape of the objects may be altered by the user. Such a scheme can make a useful addition to an interactive design package in a CG/CAD/CAM/CAE environment. It provides the users complete control over the curve segments and surface patches to modify the shape to achieve a model object. The changes will be local and that the shape will change in a stable manner. The scheme is quite simple, easy to implement and computationally economical as compared to its cubic and bi-cubic counter parts. The authors are thinking to extend the scheme for various applications including font designing, image outline capture, modeling animation paths, and others.

\section{Acknowledgements}

This work was supported by Kuwait University.

\section{REFERENCES}

[1] J. A. Gregory, and P.K. Yuen, An arbitrary mesh network scheme using rational splines, in: T. Lyche and L.L. Schumaker (eds.), Mathematical Methods in Computer Aided Geometric Design II, Academic Press, 321-329, 1992.

[2] J. A. Gregory, M. Sarfraz, and P.K. Yuen, Curves and Surfaces for Computer Aided Design using Rational Cubic Splines, Engineering with Computers, 11:94-102, 1995.

[3] J. Hoschek, Circular splines, Computer-Aided Design, 24:611-618, 1992.

[4] M. Sarfraz, M. Hussain, and Z. Habib, Local convexity preserving rational cubic spline curves, Proceedings of 
IEEE Conference on Information Visualization, IV'97, London, 211-218, 1997.

[5] T. A. Foley and H. S. Ely, Interpolation with interval and point tension controls using cubic weighted $\mathrm{Nu}$-splines, ACM Transactions on Mathematical Software, 13(1): 68-96, 1987.

[6] L. Piegl, and W. Tiller, The NURBS Book, Springer, 1995.

[7] Sarfraz, M., Al-Mulhem, M., Al-Ghamdi, J., and Hussain, A., Quadratic Representation to a $C^{1}$ Rational Cubic Spline with Interval Shape Control, Proc International Conference on Imaging Science, Systems, and Technology (CISST'98), USA, 322-329, 1998.

[8] J. S. Kouh, and S. W. Chau, Computer-aided geometric design and panel generation for hull forms based on rational cubic Bezier curves, , Computer Aided Geometric Design, 10:537-549, 1993.

[9] V. Pratt, Techniques for conic splines, Proceedings of SIGGRAPH, 151-159, 1985.

[10] T. Pavlidis, Curve fitting with conic splines, ACM Transactions on Graphics, 1-31, 1983.

[11] M. Plass and Maureen Stone, Curve-fitting with Piecewise Parametric Cubics, Computer Graphics, 17(3): 229-239, 1983.

[12] G. Nielson, Rectangular Nu-splines, IEEE Computer Graphics and Applications, 35-40, 1986.

[13] J. A. Gregory and M. Sarfraz, A rational cubic spline with tension, Computer Aided Geometric Design, 7:1-13, 1990.

[14] T. A. Foley and H. S. Ely, Interpolation with interval and point tension controls using cubic weighted Nu-splines, ACM Transactions on Mathematical Software, 13(1): 68-96, 1987.

[15] M. Paluszny and R. Patterson, A family of tangent continous cubic algebraic splines, ACM Transactions on Graphics, 12(3):209-232, 1993.

[16] J. C. Beatty R. Bartels and K. S. Booth, Experimental comparision of splines using the shape-matching paradigm, ACM Transactions on Graphics, 12(3):179-208, 1993.
[17] B. A. Barsky, Computer Graphics and Geometric Modeling using Beta-Splines, Springer-verlag, 1986, Tokyo.

[18] T. N. T. Goodman, Properties of Beta-Splines, Journal of Approximation Theory, 44(2):132-153, 1985.

[19] B. Barsky and J. Beatty, Local control of bias and tension in Beta-Splines, ACM Transactions on Graphics, 2(2):73-77, 1983.

[20] D. Joe, Multiple knot and rational cubic beta-splines, ACM Transactions on Graphics, 8(2):100-120, 1989.

[21] T. N. T. Goodman and K. Unsworth, Manipulating shape and producing geometric continuity in beta-splines curves, IEEE Computer Graphics and Applications, 6(2):50-56, 1986.

[22] M. Sarfraz, Interactive curve modeling with applications to computer graphics, vision and image processing. Springer, 2008.

[23] M. Sarfraz, M. Hussain, M. Irshad, and A. Khalid, Approximating boundary of bitmap characters using genetic algorithm, Seventh International Conference on Computer Graphics, Imaging and Visualization (CGIV'10), 2010, 671-680.

[24] Z.R. Yahya, A.R.M Piah and A.A. Majid, $\mathrm{G}^{1}$ continuity conics for curve fitting using particle swarm optimization, E. Banissi et al. (Eds.) 15th International Conference on Information Visualization,. IV, 2011, 497-501.

[25] M. Sarfraz, S. Raza and M. Baig, Capturing image outlines using soft computing approach with conic splines, International Conference of Soft Computing and Pattern Recognition, 2009, 289-294.

[26] P. Priza, S. M. Shamsuddin and A. Ali, Differential evolution optimization for Bezier curve fitting, Seventh International Conference on Computer Graphics, Imaging and Visualization, 2010, 68-72.

[27] M. Sarfraz, M. Al-Mulhem, J. Al-Ghamdi, and A. M. Hussain, Representing a $C^{1}$ Rational Quadratic Spline with Interval Shape Control, Proceedings of International Conference on Imaging Science, Systems, and Technology (CISST'98), Las Vegas, Nevada, USA, CSREA Press, USA, 322-329, 1998. 